\title{
Optimality Conditions for Quadratic Programming Problems in Hilbert Spaces
}

\author{
$\mathrm{Vu}$ Van Dong
}

\begin{abstract}
In this paper, we give optimality conditions for the quadratic programming problems with constraints defined by finitely many convex quadratic constraints in Hilbert spaces. As special cases, we obtain optimality conditions for the quadratic programming problems under linear constraints in Hilbert spaces.
\end{abstract}

\section{Introduction}

Let $\mathcal{H}$ be a real Hilbert space with inner product $\langle\cdot, \cdot\rangle$ and the induced norm $\|\cdot\|$. In this paper we will study the following optimization problem

$(\mathrm{QCQP})$

$$
\begin{gathered}
\min f(x):=\frac{1}{2}\langle x, Q x\rangle+\langle c, x\rangle \\
\text { s.t. } \quad x \in \mathcal{H}: g_{i}(x):=\frac{1}{2}\left\langle x, Q_{i} x\right\rangle+\left\langle c_{i}, x\right\rangle+\alpha_{i} \leq 0, \quad i=1,2, \ldots, m,
\end{gathered}
$$

where $\mathcal{H}$ is a Hilbert space, $Q: \mathcal{H} \rightarrow \mathcal{H}$ is a continuous linear self-adjoint operator, $Q_{i}$ is positive semidefinite continuous linear self-adjoint operator on $\mathcal{H}, c, c_{i} \in \mathcal{H}$, and $\alpha_{i}$ are real numbers, $i=1,2, \ldots, m$.

The constraint set of $\mathrm{QCQP})$ is denoted by

$$
F=\left\{x \in \mathcal{H} \mid g_{i}(x)=\frac{1}{2}\left\langle x, Q_{i} x\right\rangle+\left\langle c_{i}, x\right\rangle+\alpha_{i} \leq 0 \text { for all } i=1, \ldots, m\right\} .
$$

If $Q_{i}$ are zero operators for all $i=1, \ldots, m$, then we say that QCQP is a quadratic programming problem under linear constraints and denote it by (QPL). Note that if $Q$ and $Q_{i}$ are zero operators for all $i=1, \ldots, m$, then $\mathrm{QCQP}$ becomes a linear programming problem and will be denoted by (LP).

Quadratic programming problems (QP problems, in short) have been studied fairly complete in the setting of Euclidean spaces, see [8] and references therein. For infinite dimensional spaces, it was extended to Hilbert spaces. Existence of the solutions for

Received September 3, 2020; Accepted March 7, 2021.

Communicated by Jein-Shan Chen.

2020 Mathematics Subject Classification. 90C20, 90C26, 90C30.

Key words and phrases. quadratic programming, Hilbert spaces, convex quadratic constraints, optimality condition, tangent cone. 
QP problems in Hilbert spaces have been investigated extensively in various versions, see [1, 3, 4, 7, 10 12] and references therein.

Optimality conditions for nonlinear programming have been intensively studied in literatures, such as 6, 6] and therein reference. Optimality conditions for (QCQP are wellknown in [2]. For first and second-order necessary conditions, the proof techniques and the conclusions are the same for the finite-dimensional and the infinite-dimensional situations. However, this changes completely when trying to establish sufficient optimality condition. Borwein 2] gave the second-order sufficient condition by assuming that constraint set $F$ is finite-dimensional. Bonnans and Shapiro [1, Theorem 3.130] gave necessary and sufficient conditions for the quadratic programming problems under linear constraints in Hilbert spaces.

The purpose of this paper is to give optimality conditions for the quadratic programming problems with constraints defined by finitely many convex quadratic constraints in Hilbert spaces. Our result is established without requesting finiteness dimension of constraint set.

This paper is organized as follows. Some preliminaries are given in Section 2 , Section 3 is devoted to discuss the first-order optimality conditions for QCQP. Second-order optimality conditions for QQQP are derived in Section 4 . Finally, we conclude the paper by emphasizing the results that have been obtained.

\section{Notations and preliminary results}

In this section we recall some notations and known results which will be used in our analysis. For details, we refer to [1].

In this paper, $\operatorname{dist}(x, S)=\inf _{y \in S}\|x-y\|$ stands for the distance from the point $x \in \mathcal{H}$ to set $S \subset \mathcal{H}$. The norm of a continuous linear operator $Q: \mathcal{H} \rightarrow \mathcal{H}$ shall be defined $\|Q\|=\sup \left\{\frac{\|Q x\|}{\|x\|} \mid x \neq 0\right\}$.

The following cones will be important for the formulation of our optimality conditions.

Definition 2.1. (see, e.g., [1, p. 45]) Let $x \in F$ be a feasible point of problem (QCQP) and denote by $I(x)=\left\{i \in\{1,2, \ldots, m\} \mid g_{i}(x)=0\right\}$ the set of inequality constraints active at $x$, as well as by

$$
T_{F}(x)=\{h \in \mathcal{H} \mid \operatorname{dist}(x+t h, F)=o(t), t \geq 0\}
$$

where $r(t)=o(t)$ mean that $\frac{r(t)}{t} \rightarrow 0$ as $t \rightarrow 0$, the tangent cone of $F$ at $x$. Later on, we will also use the critical cone of the problem $(\overline{Q C Q P})$ at $x$ :

$$
C(x)=\left\{h \in T_{F}(x) \mid\langle Q x+c, h\rangle=0\right\} .
$$


Finally, the radial cone to $F$ at $x$ is

$$
\mathcal{R}_{F}(x)=\left\{h \in \mathcal{H} \mid \exists t^{*}>0, \forall t \in\left[0, t^{*}\right], x+t h \in F\right\}
$$

Definition 2.2. (Mangasarian-Fromovitz constraint qualification, see, e.g., [1, p. 71]) Consider problem $\mathrm{QCQP}$. The feasible point $\bar{x}$ is called regular if

$$
\exists h \in \mathcal{H}:\left\langle Q_{i} \bar{x}+c_{i}, h\right\rangle<0, \quad \forall i \in I(\bar{x}) .
$$

Remark 2.3. Note that if $\bar{x} \in F$ is regular, then $T_{F}(\bar{x})$ is formulated as follows (see 1 , Example 3.39])

$$
T_{F}(\bar{x})=\left\{h \in \mathcal{H} \mid\left\langle Q_{i} \bar{x}+c_{i}, h\right\rangle \leq 0, \forall i \in I(\bar{x})\right\}
$$

To obtain our results, we will need the following lemma, which is an extension of a Hoffman estimate for the distance to the set of solutions to a system of linear inequalities.

Lemma 2.4. (see [5, Theorem 3]) Let $\mathcal{H}$ be a Hilbert space. Let $x_{i}^{*} \in \mathcal{H}, i=1,2, \ldots, m$, be given, and consider the set

$$
S=\left\{x \in \mathcal{H} \mid\left\langle x_{i}^{*}, x\right\rangle \leq 0, i=1,2, \ldots, m\right\} .
$$

Then there exists a constant $k>0$ such that for any $x \in \mathcal{H}$,

$$
\operatorname{dist}(x, S) \leq k\left(\sum_{i=1}^{m}\left\langle x_{i}^{*}, x\right\rangle_{+}\right)
$$

where $[a]_{+}:=\max \{a, 0\}$.

\section{First-order optimality conditions}

In this section we will establish first-order necessary and sufficient optimality conditions for $\mathrm{QCQP}$.

Theorem 3.1. Let $\bar{x}$ be a feasible point of the problem QCQP.

(i) If $\bar{x}$ is a local solution of this problem, then

$$
\langle Q \bar{x}+c, x-\bar{x}\rangle \geq 0 \quad \text { for every } x \in F .
$$

(ii) The point $\bar{x}$ is a local solution of $\mathrm{QCQP}$ if

$$
Q_{i}=0, \quad \forall i \in I(\bar{x}) \quad \text { and } \quad\langle Q \bar{x}+c, x-\bar{x}\rangle>0, \quad \forall x \in F \backslash\{\bar{x}\}
$$


Proof. (i) Let $\bar{x}$ be a local solution of QCQP. Choose $\mu>0$ such that

$$
f(y)-f(\bar{x}) \geq 0, \quad \forall y \in F \cap B(\bar{x}, \mu) .
$$

Given any $x \in F \backslash\{\bar{x}\}$. Since $F$ is a convex set, it follows that there exists $\delta>0$ such that

$$
\bar{x}+t(x-\bar{x})=t x+(1-t) \bar{x}
$$

belonging to $F \cap B(\bar{x}, \mu)$ wherever $t \in(0, \delta)$. Hence

$$
\langle Q \bar{x}+c, x-\bar{x}\rangle=\lim _{t \downarrow 0} \frac{f(\bar{x}+t(x-\bar{x}))-f(\bar{x})}{t} \geq 0 \quad \text { for every } x \in F .
$$

Property (3.1) has been established.

(ii) On the contrary, suppose that $(3.2)$ is valid, $\bar{x}$ is not a local minimum for QCQP. Then there exists a sequence of feasible points $x_{k}$, converging to $\bar{x}$, such that

$$
f\left(x_{k}\right)<f(\bar{x}) \text { for all } k \text {. }
$$

The sequence $\left\{\frac{x_{k}-\bar{x}}{\left\|x_{k}-\bar{x}\right\|}\right\}$ is bounded and hence it has a weakly convergent subsequence. There is no loss of generality in assuming that the sequence $\left\{\frac{x_{k}-\bar{x}}{\left\|x_{k}-\bar{x}\right\|}\right\}$ converges weakly to some $h \in \mathcal{H}$. We have

$$
f\left(x_{k}\right)-f(\bar{x})=\left\langle Q \bar{x}+c, x_{k}-\bar{x}\right\rangle+\frac{1}{2}\left\langle x_{k}-\bar{x}, Q\left(x_{k}-\bar{x}\right)\right\rangle<0, \quad \forall k .
$$

Diving the last inequality by $\left\|x_{k}-\bar{x}\right\|$ and taking the limits as $k \rightarrow \infty$, we obtain

$$
\langle Q \bar{x}+c, h\rangle \leq 0
$$

Since $Q_{i}=0, \forall i \in I(\bar{x})$,

$$
g_{i}\left(x_{k}\right)-g_{i}(\bar{x})=\left\langle c_{i}, x_{k}-\bar{x}\right\rangle \leq 0, \quad \forall i \in I(\bar{x}) .
$$

Therefore $\left\langle c_{i}, h\right\rangle=\lim _{k \rightarrow \infty}\left\langle c_{i}, \frac{x_{k}-\bar{x}}{\left\|x_{k}-\bar{x}\right\|}\right\rangle \leq 0, \forall i \in I(\bar{x})$ and hence $g_{i}(\bar{x}+t h) \leq 0$ for every $i \in I(\bar{x})$ and $t>0$. Obviously, there exists $t^{*}>0$ such that $g_{i}(\bar{x}+t h) \leq 0$ for every $i \notin I(\bar{x})$ and $t \in\left(0, t^{*}\right)$. Consequently, $\bar{x}+t h \in F$ for every $t \in\left(0, t^{*}\right)$. Substituting $x=\bar{x}+$ th into 3.2 gives $\langle Q \bar{x}+c, h\rangle>0$ which contradicts $(3.3)$.

The proof is complete.

The following example shows that (3.1) is necessary but not sufficient for $\bar{x}$ to be a local solution of QCQP.

Example 3.2. Consider the programming problem

$$
\min f(x)=\frac{1}{2}\langle x, Q x\rangle \text { subject to } x \in \mathbb{R}^{2}: g_{1}(x)=\left\langle c_{1}, x\right\rangle+\alpha_{1} \leq 0,
$$


where $Q: \mathbb{R}^{2} \rightarrow \mathbb{R}^{2}$ is defined by $Q x=\left(-x_{1}, 0\right), c_{1}=(1,0)$ and $\alpha_{1}=-1$.

Let

$$
F=\left\{x=\left(x_{1}, x_{2}\right) \in \mathbb{R}^{2} \mid g_{1}(x) \leq 0\right\}=\left\{x=\left(x_{1}, x_{2}\right) \in \mathbb{R}^{2} \mid x_{1}-1 \leq 0\right\} .
$$

For $\bar{x}=(0,1)$ we have $\bar{x}=(0,1) \in F$ and $Q \bar{x}=0$. It follows that the condition (3.1) is satisfied.

Taking $x^{\varepsilon}=(\varepsilon, 1)$, where $\varepsilon$ is a positive number such that $\varepsilon<1$, we have $x^{\varepsilon} \in F$ and

$$
f\left(x^{\varepsilon}\right)=-\frac{\varepsilon^{2}}{2}<0=f(\bar{x}) .
$$

Hence $\bar{x}=(0,1)$ is not a local solution of $(3.4)$.

The following example shows that the assumption $Q_{i}=0, \forall i \in I(\bar{x})$ cannot be dropped from assumption of Theorem 3.1(ii).

Example 3.3. Consider the following programming problem

$$
\begin{array}{ll} 
& \min f(x)=\frac{1}{2}\langle x, Q x\rangle+\langle c, x\rangle \\
\text { s.t. } & x \in \mathbb{R}^{3}: g_{1}(x)=\frac{1}{2}\left\langle x, Q_{1} x\right\rangle \leq 0, g_{2}(x)=\left\langle c_{2}, x\right\rangle+\alpha_{2} \leq 0,
\end{array}
$$

where $Q: \mathbb{R}^{3} \rightarrow \mathbb{R}^{3}$ is defined by $Q x=\left(-x_{1}, 0,0\right), Q_{1}: \mathbb{R}^{3} \rightarrow \mathbb{R}^{3}$ is defined by $Q_{1} x=$ $\left(0, x_{2}-x_{3},-x_{2}+x_{3}\right), c=(0,1,0), c_{2}=(0,-1,0)$ and $\alpha_{2}=1$.

For $\bar{x}=(0,1,1)$ we have $\bar{x} \in F, g_{1}(\bar{x})=0, Q_{1} \neq 0$ and

$$
\langle Q \bar{x}+c, x-\bar{x}\rangle=x_{2}-1>0, \quad \forall x \in F \backslash\{\bar{x}\} .
$$

Taking $x^{\varepsilon}=(\varepsilon, 1,1)$, where $\varepsilon$ is a positive number such that $\varepsilon<1$, we have $x^{\varepsilon} \in F$ and

$$
f\left(x^{\varepsilon}\right)=-\frac{\varepsilon^{2}}{2}+1<1=f(\bar{x}) .
$$

Hence $\bar{x}=(0,1,1)$ is not a local solution of $(3.5)$.

The following example is constructed to show that 3.2 can guarantee $\bar{x}$ to be a local solution, but it is not a global solution.

Example 3.4. Consider the following programming problem

$$
\begin{array}{cl} 
& \min f(x)=\frac{1}{2}\langle x, Q x\rangle+\langle c, x\rangle \\
\text { s.t. } & x \in \mathbb{R}^{2}: g_{1}(x)=\left\langle c_{1}, x\right\rangle \leq 0, g_{2}(x)=\left\langle c_{1}, x\right\rangle \leq 0,
\end{array}
$$

where $Q: \mathbb{R}^{2} \rightarrow \mathbb{R}^{2}$ is defined by $Q x=\left(x_{1},-9 x_{2}\right), c=(-1,3), c_{1}=(1,-2)$ and $c_{2}=$ $(0,-1)$. 
Let

$$
F=\left\{x \in \mathbb{R}^{2} \mid g_{1}(x) \leq 0, g_{2}(x) \leq 0\right\}
$$

Taking $\bar{x}=(0,0)$, we have $\bar{x} \in F$. It is a simple matter to check that

$$
\langle Q \bar{x}+c, x-\bar{x}\rangle=\langle c, x\rangle=-x_{1}+3 x_{2} \geq x_{2}>0, \quad \forall x \in F \backslash\{\bar{x}\}
$$

Let $\varepsilon$ be a positive number such that $\varepsilon<1 / 2$ and let $U_{\bar{x}}^{\varepsilon}$ be neighborhood of $\bar{x}$. Put $\mathcal{N}_{\bar{x}}=U_{\bar{x}}^{\varepsilon} \cap F$. Then, for all $x \in \mathcal{N}_{\bar{x}}$ we have

$$
f(x)=\frac{1}{2}\left(x_{1}-3 x_{2}\right)\left(x_{1}+3 x_{2}-2\right) \geq 0=f(0)
$$

for all $x \in \mathcal{N}_{\bar{x}}$. Hence $\bar{x}=(0,0)$ is a local minimum of 3.6 .

Note that if $\widehat{x}=(0,1)$, then $\widehat{x} \in F$ and $f(\widehat{x})=-\frac{3}{2}<f(\bar{x})=0$. Therefore $\bar{x}$ is not a global solution of 3.6 .

Remark 3.5. If $Q$ is a positive semidefinite continuous linear self-adjoint operator, then (3.1) is sufficient condition for $\bar{x}$ to be a global solution of QCQP. Indeed, by positive semi-definiteness of $Q$, it follows that $f(x)$ is a convex function. For every $x \in F$ we have

$$
0 \leq\langle Q \bar{x}+c, x-\bar{x}\rangle \leq f(x)-f(\bar{x})
$$

Therefore $\bar{x}$ is a global solution of QCQP.

The following theorem is just a special case of nonlinear programming with smooth data. However, for the sake of completeness, we give the complete proof here.

Theorem 3.6. If $\bar{x} \in \mathcal{H}$ is a local solution of problem (QCQP and if $\bar{x}$ is regular, then there exists $\lambda=\left(\lambda_{1}, \ldots, \lambda_{m}\right) \in \mathbb{R}^{m}$ such that

$$
\left\{\begin{array}{l}
Q \bar{x}+c+\sum_{i=1}^{m} \lambda_{i}\left(Q_{i} \bar{x}+c_{i}\right)=0 \\
\frac{1}{2}\left\langle\bar{x}, Q_{i} \bar{x}\right\rangle+\left\langle c_{i}, \bar{x}\right\rangle+\alpha_{i} \leq 0 \\
\lambda_{i}\left(\frac{1}{2}\left\langle\bar{x}, Q_{i} \bar{x}\right\rangle+\left\langle c_{i}, \bar{x}\right\rangle+\alpha_{i}\right)=0 \\
\lambda_{i} \geq 0, \quad i=1, \ldots, m
\end{array}\right.
$$

Proof. Suppose that $\bar{x} \in \mathcal{H}$ is a local solution of problem QCQP and $\bar{x}$ is regular. Then, by regularity of $\bar{x}$ we have

$$
T_{F}(\bar{x})=\left\{h \in \mathcal{H} \mid\left\langle Q_{i} \bar{x}+c_{i}, h\right\rangle \leq 0, \forall i \in I(\bar{x})\right\}
$$

It follows from [1, Lemma 3.7] that $h=0$ is an optimal solution of the linearized problem

$$
\min _{h \in \mathcal{H}}\langle Q \bar{x}+c, h\rangle \quad \text { subject to } \quad\left\langle Q_{i} \bar{x}+c_{i}, h\right\rangle \leq 0, \quad i \in I(\bar{x}) .
$$


The problem (3.8) is a linear programming problem with a finite (equal zero) optimal value. By Theorem 2.202 in [1], we have that the set of optimal solution of the dual problem of 3.8

$$
\max _{\lambda_{i} \geq 0} 0 \quad \text { subject to } Q \bar{x}+c+\sum_{i \in I(\bar{x})} \lambda_{i}\left(Q_{i} \bar{x}+c_{i}\right)=0
$$

is nonempty.

Put $\lambda_{i}=0$ for all $i \in I \backslash I(\bar{x})$ (where $I=\{1,2, \ldots, m\}$ ), and $\lambda=\left(\lambda_{1}, \ldots, \lambda_{m}\right)$. From 3.9 we obtain the first equality in (3.7). Since $\bar{x} \in F$ and $\lambda_{i}\left(\frac{1}{2}\left\langle\bar{x}, Q_{i} \bar{x}\right\rangle+\left\langle c_{i}, \bar{x}\right\rangle+\alpha_{i}\right)=0$ for each $i \in I$, the other conditions in (3.7) are satisfied too. The proof is complete.

Remark 3.7. If $\bar{x} \in \mathcal{H}$ is a local solution of problem QCQP and if $\bar{x}$ is regular, then (3.7) is equivalent to following condition

$$
\langle Q \bar{x}+c, h\rangle \geq 0 \quad \text { for all } h \in T_{F}(\bar{x}) .
$$

Indeed, 3.10 implies 3.7) follows immediately from the proof of Theorem 3.6. It remains to prove that (3.7) implies (3.10). Suppose that (3.7) is satisfied. Then, for every $h \in T_{F}(\bar{x})$ we have $\left\langle Q_{i} \bar{x}+c_{i}, h\right\rangle \leq 0$ for all $i \in I(\bar{x})$ and

$$
\langle Q \bar{x}+c, h\rangle=-\sum_{i \in I(\bar{x})} \lambda_{i}\left\langle Q_{i} \bar{x}+c_{i}, h\right\rangle \geq 0 .
$$

Hence 3.10 is satisfied.

The following example shows that the conclusion of Theorem 3.6 fails if the assumption on the regularity of $\bar{x}$ is omitted.

Example 3.8. Consider the programming problem

$$
\begin{gathered}
\min f(x)=\frac{1}{2}\langle x, Q x\rangle+\langle c, x\rangle \\
\text { subject to } \quad x=\left(x_{1}, x_{2}\right) \in \mathbb{R}^{2}: g_{1}(x)=\frac{1}{2}\left\langle x, Q_{1} x\right\rangle \leq 0,
\end{gathered}
$$

where $Q: \mathbb{R}^{2} \rightarrow \mathbb{R}^{2}$ is defined by $Q x=\left(x_{1}, 0\right), c=(0,-1)$ and $Q_{1}: \mathbb{R}^{2} \rightarrow \mathbb{R}^{2}$ is defined by $Q_{1} x=\left(x_{1}-x_{2},-x_{1}+x_{2}\right)$.

Let $F=\left\{x=\left(x_{1}, x_{2}\right) \in \mathbb{R}^{2} \mid g_{1}(x) \leq 0\right\}$. We have

$$
F=\left\{x=\left(x_{1}, x_{2}\right) \in \mathbb{R}^{2} \mid\left(x_{1}-x_{2}\right)^{2} \leq 0\right\} .
$$

For $\bar{x}=(1,1) \in \mathbb{R}^{2}$ we have $\bar{x} \in F$ and $g_{1}(\bar{x})=0$. Since $\left\langle Q_{1} \bar{x}, h\right\rangle=0$ for all $h \in \mathbb{R}^{2}$, we have $\bar{x}$ is irregular.

Since $x_{1}=x_{2}$, we have $f(x)=\frac{1}{2} x_{1}^{2}-x_{1}=\frac{1}{2}\left(x_{1}-1\right)^{2}-\frac{1}{2} \geq-\frac{1}{2}$ for all $x=\left(x_{1}, x_{2}\right) \in F$. It follows that $\bar{x}=(1,1)$ is a local solution of 3.11 . Since $Q \bar{x}+c=(1,-1)$ and $Q_{1} \bar{x}=0$, we see that there exists no $\lambda_{1} \geq 0$ such that $Q \bar{x}+c+\lambda_{1} Q_{1} \bar{x}=0$. Hence the first equality in (3.7) does not hold. 


\section{Second-order optimality conditions}

In this section, we shall establish second-order necessary and sufficient condition for $\bar{x}$ to be (a strict) a local solution of problem QCQP. For this, we will need the following assumption

(H) $\left(h \in T_{F}(\bar{x}),\langle Q \bar{x}+c, h\rangle=0, \quad\left\langle Q_{i} \bar{x}+c_{i}, h\right\rangle=0\right) \quad \Longrightarrow \quad\left(\left\langle h, Q_{i} h\right\rangle=0\right), \quad \forall i \in I(\bar{x})$.

It is easily seen that the assumption $(\sqrt{\mathrm{H}})$ is satisfied if one of the following conditions holds:

(i) $Q_{i}=0$ for all $i \in I(\bar{x})$,

(ii) $\langle Q \bar{x}+c, h\rangle>0$ for all $h \in T_{F}(\bar{x}) \backslash\{0\}$,

(iii) $\left\langle Q_{i} \bar{x}+c_{i}, h\right\rangle<0$ for all $h \in T_{F}(\bar{x}) \backslash\{0\}$ and for all $i \in I(\bar{x})$.

Theorem 4.1. Let $\bar{x}$ be a feasible point of the problem (QCQP and let $\bar{x}$ be regular. Suppose that the assumption $(\mathrm{H})$ is satisfied. Then, $\bar{x}$ is a local solution of (QCQP if and only if the following two conditions are satisfied:

$$
\begin{gathered}
\langle Q \bar{x}+c, h\rangle \geq 0 \text { for all } h \in T_{F}(\bar{x}), \\
\text { if } h \in T_{F}(\bar{x}) \text { and }\langle Q \bar{x}+c, h\rangle=0 \text { then }\langle h, Q h\rangle \geq 0 .
\end{gathered}
$$

Proof. Since $\bar{x}$ is regular, it follows from Remark 2.3 that

$$
T_{F}(\bar{x})=\left\{h \in \mathcal{H} \mid\left\langle Q_{i} \bar{x}+c_{i}, h\right\rangle \leq 0, \forall i \in I(\bar{x})\right\}
$$

Necessity. By Remark 3.7, assertion (4.1) holds.

Suppose that there exists $h \in T_{F}(\bar{x})$ such that $\langle Q \bar{x}+c, h\rangle=0$ but $\langle h, Q h\rangle<0$. Let us first show that there exists $t^{*}>0$ such that

$$
\bar{x}+t h \in F, \quad \forall t \in\left(0, t^{*}\right) .
$$

For $i \in I(\bar{x})$, we have $g_{i}(\bar{x})=0$. Since $\left\langle Q_{i} \bar{x}+c_{i}, h\right\rangle \leq 0$ and by the assumption $(\overline{\mathrm{H}})$, there exists $t_{1}^{*}>0$ such that

$$
g_{i}(\bar{x}+t h)=g_{i}(\bar{x})+t\left\langle Q_{i} \bar{x}+c_{i}, h\right\rangle+\frac{t^{2}}{2}\left\langle h, Q_{i} h\right\rangle \leq 0, \quad \forall t \in\left(0, t_{1}^{*}\right) .
$$

For $i \notin I(\bar{x})$ we have $g_{i}(\bar{x})<0$. Since $g_{i}(\bar{x}+t h)=g_{i}(\bar{x})+t\left\langle Q_{i} \bar{x}+c_{i}, h\right\rangle+\frac{t^{2}}{2}\left\langle h, Q_{i} h\right\rangle$ is a quadratic function (in the variable $t$ ) with $g_{i}(\bar{x})<0$ and $\frac{1}{2}\left\langle h, Q_{i} h\right\rangle \geq 0$, there exists $t_{2}^{*}>0$ such that

$$
g_{i}(\bar{x}+t h) \leq 0 \quad \text { for all } t \in\left(0, t_{2}^{*}\right)
$$


Let $t^{*}=\min \left\{t_{1}^{*}, t_{2}^{*}\right\}$. From 4.4 and 4.5 we obtain 4.3.

Consequently,

$$
f(\bar{x}+t h)-f(\bar{x})=t\langle Q \bar{x}+c, h\rangle+\frac{t^{2}}{2}\langle h, Q h\rangle=\frac{t^{2}}{2}\langle h, Q h\rangle<0, \quad \forall t \in\left(0, t^{*}\right) .
$$

This contradicts our assumption that $\bar{x}$ is a local solution of QCQP. Hence, assertion 4.2 holds.

Sufficiency. On the contrary, suppose that the conditions (4.1), 4.2) are satisfied, but $\bar{x}$ is not a locally optimal solution of QCQP. Then there exists a sequence of feasible points $x_{k}$, converging to $\bar{x}$ such that

$$
f\left(x_{k}\right)<f(\bar{x}) \text { for all } k \text { large enough. }
$$

Set $t_{k}:=\left\|x_{k}-\bar{x}\right\|$ and $h_{k}:=\frac{x_{k}-\bar{x}}{t_{k}}$. We have $t_{k}>0,\left\|h_{k}\right\|=1$ and

$$
\left\langle Q_{i} \bar{x}+c_{i}, h_{k}\right\rangle=\frac{1}{t_{k}}\left\{g_{i}\left(x_{k}\right)-g_{i}(\bar{x})-\frac{1}{2}\left\langle x_{k}-\bar{x}, Q_{i}\left(x_{k}-\bar{x}\right)\right\rangle\right\} \leq 0 \quad \text { for } i \in I(\bar{x}) .
$$

Put $C(\bar{x})=\left\{h \in \mathcal{H} \mid\langle Q \bar{x}+c, h\rangle=0,\left\langle Q_{i} \bar{x}+c_{i}, h\right\rangle \leq 0, i \in I(\bar{x})\right\}$. It follows from Lemma 2.4 that

$$
\operatorname{dist}\left(h_{k}, C(\bar{x})\right) \leq \beta\left(\left[\left\langle Q \bar{x}+c, h_{k}\right\rangle\right]_{+}+\sum_{i \in I(\bar{x})}\left[\left\langle Q_{i} \bar{x}+c_{i}, h_{k}\right\rangle\right]_{+}\right)=\beta\left(\left[\left\langle Q \bar{x}+c, h_{k}\right\rangle\right]_{+}\right),
$$

where $\beta>0$ depends on $Q \bar{x}+c$ and $Q_{i} \bar{x}+c_{i}$.

By (4.6) and

$$
f\left(x_{k}\right)-f(\bar{x})=t_{k}\left\langle Q \bar{x}+c, h_{k}\right\rangle+\frac{t_{k}^{2}}{2}\left\langle h_{k}, Q h_{k}\right\rangle,
$$

it follows that

$$
t_{k}\left\langle Q \bar{x}+c, h_{k}\right\rangle<-\frac{t_{k}^{2}}{2}\left\langle h_{k}, Q h_{k}\right\rangle
$$

Since $\left|\left\langle h_{k}, Q h_{k}\right\rangle\right| \leq\|Q\|\left\|h_{k}\right\|^{2}=\|Q\|$, it follows that $-\frac{t_{k}}{2}\left\langle h_{k}, Q h_{k}\right\rangle \rightarrow 0$ as $k \rightarrow \infty$. Combining this with 4.7) we have that $t_{k}\left(\left\langle Q \bar{x}+c, h_{k}\right\rangle\right) \leq o\left(t_{k}\right)$, and hence there exists a critical direction $\widehat{h}_{k} \in C(\bar{x})$ such that $\widehat{h}_{k}-h_{k} \rightarrow 0$, and hence $\left\|\widehat{h}_{k}\right\|=1$.

Observe that

$$
\left\langle\widehat{h}_{k}, Q \widehat{h}_{k}\right\rangle-\left\langle h_{k}, Q h_{k}\right\rangle=\left\langle\widehat{h}_{k}+h_{k}, Q\left(\widehat{h}_{k}-h_{k}\right)\right\rangle \leq\left\|\widehat{h}_{k}+h_{k}\right\|\|Q\|\left\|\widehat{h}_{k}-h_{k}\right\| .
$$

From this and $\left\|\widehat{h}_{k}-h_{k}\right\| \leq \beta\left(\left[\left\langle Q \bar{x}+c, h_{k}\right\rangle\right]_{+}\right)$we deduce that

$$
\left\langle\widehat{h}_{k}, Q \widehat{h}_{k}\right\rangle-\left\langle h_{k}, Q h_{k}\right\rangle \leq 2 \beta\|Q\|\left(\left[\left\langle Q \bar{x}+c, h_{k}\right\rangle\right]_{+}\right) .
$$


Consequently, since for the function $f(\cdot)$ the second order Taylor expansion is exact, we have by 4.8 that

$$
\begin{aligned}
f\left(x_{k}\right) & =f(\bar{x})+t_{k}\left\langle Q \bar{x}+c, h_{k}\right\rangle+\frac{t_{k}^{2}}{2}\left\langle h_{k}, Q h_{k}\right\rangle \\
& \geq f(\bar{x})+\left(t_{k}\left\langle Q \bar{x}+c, h_{k}\right\rangle-t_{k}^{2} \beta\|Q\|\left(\left[\left\langle Q \bar{x}+c, h_{k}\right\rangle\right]_{+}\right)\right)+\frac{t_{k}^{2}}{2}\left\langle\widehat{h}_{k}, Q \widehat{h}_{k}\right\rangle .
\end{aligned}
$$

Since $\langle Q \bar{x}+c, h\rangle \geq 0$ for all $h \in T_{F}(\bar{x})$, we have that $\left\langle Q \bar{x}+c, h_{k}\right\rangle \geq 0$ for $k$ large enough. Hence for $k$ large enough, we have

$$
f\left(x_{k}\right)-f(\bar{x}) \geq\left(t_{k}\left\langle Q \bar{x}+c, h_{k}\right\rangle-t_{k}^{2} \beta\|Q\|\left(\left[\left\langle Q \bar{x}+c, h_{k}\right\rangle\right]_{+}\right)\right)+\frac{t_{k}^{2}}{2}\left\langle\widehat{h}_{k}, Q \widehat{h}_{k}\right\rangle \geq 0,
$$

a contradiction with 4.6 . The proof is complete.

The following example shows that Theorem 4.1 is an extension of Theorem 3.130 in 1 ] for the quadratic programming problems in Hilbert spaces.

Example 4.2. Let $\ell^{2}$ denote the Hilbert space of all square summable real sequence, $\ell^{2}=\left\{x=\left(x_{1}, x_{2}, \ldots, x_{n}, \ldots\right) \mid \sum_{n=1}^{\infty} x_{n}^{2}<\infty, x_{n} \in \mathbb{R}, n=1,2, \ldots\right\}$. The scalar product and the norm in $\ell^{2}$ are defined, respectively, by

$$
\langle x, y\rangle=\sum_{n=1}^{\infty} x_{n} y_{n}, \quad\|x\|=\left(\sum_{n=1}^{\infty} x_{n}^{2}\right)^{1 / 2} .
$$

Consider the following programming problem

$$
\begin{aligned}
& \min f(x)=\frac{1}{2}\langle x, Q x\rangle+\langle c, x\rangle \\
\text { s.t. } & x \in \ell^{2}: g_{1}(x)=\frac{1}{2}\left\langle x, Q_{1} x\right\rangle+\left\langle c_{1}, x\right\rangle \leq 0, g_{2}(x)=\left\langle c_{2}, x\right\rangle \leq 0,
\end{aligned}
$$

where $Q: \ell^{2} \rightarrow \ell^{2}$ is defined by $Q x=\left(x_{1},-x_{2}, x_{3}, \ldots\right), Q_{1}: \ell^{2} \rightarrow \ell^{2}$ is defined by $Q_{1} x=$ $\left(x_{1}, 0,0, \ldots\right), c=(-1,1,0,0, \ldots), c_{1}=(1,-1,0,0, \ldots)$ and $c_{2}=(-1,0,0, \ldots)$.

Let

$$
F=\left\{x \in \ell^{2} \mid g_{1}(x) \leq 0, g_{2}(x) \leq 0\right\} .
$$

For $\bar{x}=(0, \ldots, 0, \ldots) \in F$ and $h=\left(h_{1}, h_{2}, \ldots\right) \in \ell^{2}$. It is a simple matter to check that $\bar{x}$ is regular and

$$
\begin{aligned}
T_{F}(\bar{x}) & =\left\{h \in \ell^{2} \mid\left\langle Q_{1} \bar{x}+c_{1}, h\right\rangle \leq 0,\left\langle c_{2}, h\right\rangle \leq 0\right\} \\
& =\left\{h \in \ell^{2} \mid h_{1}-h_{2} \leq 0, h_{1} \geq 0\right\} .
\end{aligned}
$$

Since $\langle Q \bar{x}+c, h\rangle=h_{2}-h_{1}$, it follows that $\langle Q \bar{x}+c, h\rangle \geq 0$ for all $h \in T_{F}(\bar{x})$. If $h \in T_{F}(\bar{x})$ and $\langle Q \bar{x}+c, h\rangle=h_{2}-h_{1}=0$ then

$$
\langle h, Q h\rangle=h_{1}^{2}-h_{2}^{2}+h_{3}^{2}+\cdots \geq 0 .
$$


Note that if $h \in T_{F}(\bar{x}),\langle Q \bar{x}+c, h\rangle=0,\left\langle Q_{1} \bar{x}+c_{1}, h\right\rangle=0,\left\langle c_{2}, h\right\rangle=0$, then $h_{1}=h_{2}=0$ and $\left\langle h, Q_{1} h\right\rangle=h_{1}^{2}=0$. Therefore the assumption $(\mathrm{H})$ is satisfied.

Let $\varepsilon$ be a positive number such that $\varepsilon<1$ and let $V_{\bar{x}}^{\varepsilon}$ be neighborhood of $\bar{x}$. Put $\mathcal{N}_{\bar{x}}=V_{\bar{x}}^{\varepsilon} \cap F$. By taking $x^{\varepsilon}=\left(x_{1}^{\varepsilon}, x_{2}^{\varepsilon}, \ldots\right) \in \mathcal{N}_{\bar{x}}$, we have $x_{i}^{\varepsilon}<1$ for all $i=1,2, \ldots$, and $x_{2}^{\varepsilon} \geq x_{1}^{\varepsilon} \geq 0$. Since

$$
f(x)=\frac{1}{2}\left(x_{1}-x_{2}\right)\left(x_{1}+x_{2}-2\right)+\frac{1}{2} x_{3}^{2}+\frac{1}{2} x_{4}^{2}+\cdots,
$$

we have $f\left(x^{\varepsilon}\right) \geq 0=f(0)$ for all $x^{\varepsilon} \in \mathcal{N}_{\bar{x}}$. Hence $\bar{x}=(0, \ldots, 0, \ldots)$ is a local solution of (4.9).

The following example shows that the assumption $(\mathrm{H})$ cannot be dropped from the assumption of Theorem 4.1 .

Example 4.3. Consider the programming problem

$$
\begin{gathered}
\min f(x)=\frac{1}{2}\langle x, Q x\rangle+\langle c, x\rangle \\
\text { subject to } \quad x \in \mathbb{R}^{2}: g_{1}(x)=\frac{1}{2}\left\langle x, Q_{1} x\right\rangle+\left\langle c_{1}, x\right\rangle+\alpha_{1} \leq 0,
\end{gathered}
$$

where $Q: \mathbb{R}^{2} \rightarrow \mathbb{R}^{2}$ is defined by $Q x=\left(-x_{1}, 0\right), Q_{1}: \mathbb{R}^{2} \rightarrow \mathbb{R}^{2}$ is defined as $Q_{1} x=\left(x_{1}, 0\right)$, $c=(0,1), c_{1}=(0,-1)$ and $\alpha_{1}=0$.

Let $F=\left\{x \in \mathbb{R}^{2} \mid g_{1}(x) \leq 0\right\}=\left\{x \in \mathbb{R}^{2} \mid \frac{1}{2} x_{1}^{2}-x_{2} \leq 0\right\}$. Since $f(x)=-\frac{1}{2} x_{1}^{2}+x_{2} \geq$ $-\frac{1}{2} x_{1}^{2}+\frac{1}{2} x_{1}^{2}=0$, we have that $\bar{x}=(0,0) \in F$ is a local solution of 4.10).

It is easy to check that $\bar{x}$ is regular and

$$
T_{F}(\bar{x})=\left\{h \in \mathbb{R}^{2} \mid\left\langle Q_{1} \bar{x}+c_{1}, h\right\rangle \leq 0\right\}=\left\{h \in \mathbb{R}^{2} \mid-h_{2} \leq 0\right\}
$$

For $h=(1,0) \in T_{F}(\bar{x})$, we have $\langle Q \bar{x}+c, h\rangle=h_{2}=0,\left\langle Q_{1} \bar{x}+c_{1}, h\right\rangle=-h_{2}=0$ and $\left\langle h, Q_{1} h\right\rangle=1 \neq 0$. Hence the assumption $(\mathrm{H})$ do not hold. Since $\langle h, Q h\rangle=-1$, we have that the condition 4.2 does not hold.

Note that Theorem 4.1 can be reformulated in the following equivalent form which requires the use of Lagrange multipliers.

Theorem 4.4. Let $\bar{x}$ be a feasible point of the problem (QCQP and let $\bar{x}$ be regular. Suppose that the assumption $(\mathrm{H})$ is satisfied. The necessary and sufficient condition for a point $\bar{x}$ to be a local solution of (QCQP is that there exists $\lambda=\left(\lambda_{1}, \ldots, \lambda_{m}\right) \in \mathbb{R}^{m}$ such that

(a) the system (3.7) is satisfied, and 
(b) if $h \in \mathcal{H} \backslash\{0\}$ is such that $\left\langle Q_{i} \bar{x}+c_{i}, h\right\rangle=0, i \in I_{1}(\bar{x}),\left\langle Q_{i} \bar{x}+c_{i}, h\right\rangle \leq 0, i \in I_{2}(\bar{x})$, where

$$
I_{1}(\bar{x})=\left\{i: g_{i}(\bar{x})=0, \lambda_{i}>0\right\}, \quad I_{2}(\bar{x})=\left\{i: g_{i}(\bar{x})=0, \lambda_{i}=0\right\},
$$

then $\langle h, Q h\rangle \geq 0$.

Proof. Let us first prove that if $\bar{x} \in \mathcal{H}, \lambda \in \mathbb{R}^{m}$ such that the system (3.7) is satisfied and let $I_{1}(\bar{x})$ and $I_{2}(\bar{x})$ be such as in 4.11 , then

$$
\begin{aligned}
& \left\{h \in \mathcal{H} \mid\left\langle Q_{i} \bar{x}+c_{i}, h\right\rangle=0, i \in I_{1}(\bar{x}),\left\langle Q_{i} \bar{x}+c_{i}, h\right\rangle \leq 0, i \in I_{2}(\bar{x})\right\} \\
= & \left\{h \in \mathcal{H} \mid\left\langle Q_{i} \bar{x}+c_{i}, h\right\rangle \leq 0, i \in I(\bar{x}),\langle Q \bar{x}+c, h\rangle=0\right\}=C(\bar{x}) .
\end{aligned}
$$

Suppose that $h \in \mathcal{H},\left\langle Q_{i} \bar{x}+c_{i}, h\right\rangle=0, i \in I_{1}(\bar{x}),\left\langle Q_{i} \bar{x}+c_{i}, h\right\rangle \leq 0, i \in I_{2}(\bar{x})$. By (3.7) we have

$$
\begin{aligned}
\langle Q \bar{x}+c, h\rangle & =-\sum_{i=1}^{m} \lambda_{i}\left\langle Q_{i} \bar{x}+c_{i}, h\right\rangle \\
& =-\sum_{i \in I(\bar{x})} \lambda_{i}\left\langle Q_{i} \bar{x}+c_{i}, h\right\rangle-\sum_{i \notin I(\bar{x})} \lambda_{i}\left\langle Q_{i} \bar{x}+c_{i}, h\right\rangle=0 .
\end{aligned}
$$

Hence

$$
\left\{h \in \mathcal{H} \mid\left\langle Q_{i} \bar{x}+c_{i}, h\right\rangle=0, i \in I_{1}(\bar{x}),\left\langle Q_{i} \bar{x}+c_{i}, h\right\rangle \leq 0, i \in I_{2}(\bar{x})\right\} \subset C(\bar{x}) .
$$

To obtain the reverse inclusion, suppose that $h \in C(\bar{x})$. We need only to show that $\left\langle Q_{i} \bar{x}+c_{i}, h\right\rangle=0, i \in I_{1}(\bar{x})$. From (3.7) we deduce that

$$
\begin{aligned}
0 & =\langle Q \bar{x}+c, h\rangle \\
& =-\sum_{i \in I_{1}(\bar{x})} \underbrace{\lambda_{i}}_{>0}\left\langle Q_{i} \bar{x}+c_{i}, h\right\rangle-\sum_{i \in I_{2}(\bar{x})} \underbrace{\lambda_{i}}_{=0}\left\langle Q_{i} \bar{x}+c_{i}, h\right\rangle-\sum_{i \notin I(\bar{x})} \underbrace{\lambda_{i}}_{=0}\left\langle Q_{i} \bar{x}+c_{i}, h\right\rangle .
\end{aligned}
$$

Hence $\left\langle Q_{i} \bar{x}+c_{i}, h\right\rangle=0, i \in I_{1}(\bar{x})$ and

$$
\left\{h \in \mathcal{H} \mid\left\langle Q_{i} \bar{x}+c_{i}, h\right\rangle=0, i \in I_{1}(\bar{x}),\left\langle Q_{i} \bar{x}+c_{i}, h\right\rangle \leq 0, i \in I_{2}(\bar{x})\right\}=C(\bar{x}) .
$$

Necessity. Suppose that $\bar{x}$ is a local solution of QCQP and $\bar{x}$ is regular. It follows from Theorem 4.1 and Remark 3.7 that (3.7) and (4.2) hold. From (3.7), (4.2) and

$$
C(\bar{x})=\left\{h \in \mathcal{H} \mid\left\langle Q_{i} \bar{x}+c_{i}, h\right\rangle=0, i \in I_{1}(\bar{x}),\left\langle Q_{i} \bar{x}+c_{i}, h\right\rangle \leq 0, i \in I_{2}(\bar{x})\right\},
$$

it follows that (b) are satisfied.

Sufficiency. Suppose that $\bar{x} \in F$ is such that there exists $\lambda \in \mathbb{R}^{m}$ such that conditions (a) and (b) are satisfied. Then, by Remark 3.7 and

$$
\left\{h \in \mathcal{H} \mid\left\langle Q_{i} \bar{x}+c_{i}, h\right\rangle=0, i \in I_{1}(\bar{x}),\left\langle Q_{i} \bar{x}+c_{i}, h\right\rangle \leq 0, i \in I_{2}(\bar{x})\right\}=C(\bar{x}),
$$

it follows that conditions (4.1) and (4.2) are satisfied. On account of Theorem 4.1, we have $\bar{x}$ is a local solution of $\mathrm{QCQP}$. This proof is complete. 
Corollary 4.5. (cf. [1, Theorem 3.130]) Consider the quadratic programming problem under linear constraints (QPL) (i.e., (QCQP with $Q_{i}=0$ for all $i=1, \ldots, m$ ). Let $\bar{x}$ be a feasible point of the problem (QCQP. Then, the point $\bar{x}$ is a locally optimal solution of QCQP if and only if conditions (4.1) and (4.2) are satisfied.

Proof. Since $Q_{i}=0$ for all $i=1, \ldots, m$, the assumptions of Theorem 4.1 is satisfied. Hence the corollary follows.

In the remainder of this section we discuss necessary and sufficient optimality condition for $\bar{x}$ to be a strict local solution of $(\overline{Q C Q P})$. Recall that a point $\bar{x}$ is called a strict local solution of $\mathrm{QCQP}$ if there exists $\varepsilon>0$ such that

$$
f(x)>f(\bar{x}), \quad \forall x \in(F \cap B(\bar{x}, \varepsilon)) \backslash\{\bar{x}\} .
$$

Of course, if $\bar{x}$ is a strict local solution of a minimization problem then it is a local solution of that problem. The converse is not true in general.

The following theorem describes the second-order necessary and sufficient condition for a point to be a strict local solution of QCQP.

Theorem 4.6. Let $\bar{x}$ be a feasible point of the problem (QCQP and let $\bar{x}$ be regular. Suppose that the assumption $(\mathrm{H})$ is satisfied. Then, $\bar{x}$ is a strict local solution of (QCQP) if and only if the following two conditions are satisfied.

$$
\begin{gathered}
\langle Q \bar{x}+c, h\rangle \geq 0 \text { for all } h \in T_{F}(\bar{x}), \\
\text { if } h \in T_{F}(\bar{x}) \backslash\{0\} \text { and }\langle Q \bar{x}+c, h\rangle=0 \text { then }\langle h, Q h\rangle>0 \text {. }
\end{gathered}
$$

Proof. The proof of this theorem is similar to that of Theorem 4.1; for completeness we present a short proof.

Since $\bar{x}$ is regular, we have

$$
T_{F}(\bar{x})=\left\{h \in \mathcal{H} \mid\left\langle Q_{i} \bar{x}+c_{i}, h\right\rangle \leq 0, \forall i \in I(\bar{x})\right\}
$$

Necessity. By Remark 3.7, assertion 4.12 holds.

Suppose that there exists $h \in T_{F}(\bar{x}) \backslash\{0\}$ such that $\langle Q \bar{x}+c, h\rangle=0$ and $\langle h, Q h\rangle \leq 0$. Using similar arguments as in the proof of Theorem 4.1, we conclude that there exists a positive number $t^{*}$ such that

$$
\bar{x}+t h \in F, \quad \forall t \in\left(0, t^{*}\right)
$$

and

$$
f(\bar{x}+t h)-f(\bar{x})=t\langle Q \bar{x}+c, h\rangle+\frac{t^{2}}{2}\langle h, Q h\rangle=\frac{t^{2}}{2}\langle h, Q h\rangle \leq 0, \quad \forall t \in\left(0, t^{*}\right)
$$


This contradicts our the fact that $\bar{x}$ is a strict local solution of QCQP. Hence, assertion 4.13 holds.

Sufficiency. Suppose that the point $\bar{x}$ is not a strict local solution for QCQP . Then there exists a sequence of feasible points $x_{k}$, converging to $\bar{x}, x_{k} \neq \bar{x}$, such that

$$
f\left(x_{k}\right) \leq f(\bar{x}) .
$$

Set $t_{k}:=\left\|x_{k}-\bar{x}\right\|$ and $h_{k}:=\frac{x_{k}-\bar{x}}{t_{k}}$. We have $\left\langle Q_{i} \bar{x}+c_{i}, h_{k}\right\rangle \leq 0$ for $i \in I(\bar{x})$ and $k$ large enough. Then, as in the proof of Theorem 4.1, it follows by Lemma 2.4 that there there exists a critical direction $\widehat{h}_{k} \in C(\bar{x})$ such that $\widehat{h}_{k}-h_{k} \rightarrow 0,\left\|\widehat{h}_{k}\right\|=1$,

$$
\left\|\widehat{h}_{k}-h_{k}\right\| \leq \beta\left(\left[\left\langle Q \bar{x}+c, h_{k}\right\rangle\right]_{+}\right)
$$

and

$$
\left\langle\widehat{h}_{k}, Q \widehat{h}_{k}\right\rangle-\left\langle h_{k}, Q h_{k}\right\rangle \leq 2 \beta\|Q\|\left(\left[\left\langle Q \bar{x}+c, h_{k}\right\rangle\right]_{+}\right) .
$$

Consequently,

$$
\begin{aligned}
f\left(x_{k}\right) & =f(\bar{x})+t_{k}\left\langle Q \bar{x}+c, h_{k}\right\rangle+\frac{t_{k}^{2}}{2}\left\langle h_{k}, Q h_{k}\right\rangle \\
& \geq f(\bar{x})+t_{k}\left\langle Q \bar{x}+c, h_{k}\right\rangle+\frac{t_{k}^{2}}{2}\left\langle\widehat{h}_{k}, Q \widehat{h}_{k}\right\rangle-t_{k}^{2} \beta\|Q\|\left(\left[\left\langle Q \bar{x}+c, h_{k}\right\rangle\right]_{+}\right) .
\end{aligned}
$$

Since $\langle Q \bar{x}+c, h\rangle \geq 0$ for all $h \in T_{F}(\bar{x})$, we have $\left\langle Q \bar{x}+c, h_{k}\right\rangle \geq 0$ for $k$ large enough. It follows that for $k$ large enough

$$
f\left(x_{k}\right)-f(\bar{x}) \geq t_{k}\left\langle Q \bar{x}+c, h_{k}\right\rangle+\frac{t_{k}^{2}}{2}\left\langle\widehat{h}_{k}, Q \widehat{h}_{k}\right\rangle-t_{k}^{2} \beta\|Q\|\left(\left[\left\langle Q \bar{x}+c, h_{k}\right\rangle\right]_{+}\right)>0,
$$

which contradicts 4.14). The proof is complete.

Theorem 4.6 can be reformulated in the following equivalent form which requires the use of Lagrange multipliers.

Theorem 4.7. Let $\bar{x}$ be a feasible point of the problem (QCQP and let $\bar{x}$ be regular. Suppose that the assumption $(\mathrm{H})$ is satisfied. The necessary and sufficient condition for a point $\bar{x}$ to be a strict local solution of $\mathrm{QCQP}$ is that there exists $\lambda=\left(\lambda_{1}, \ldots, \lambda_{m}\right) \in \mathbb{R}^{m}$ such that

(a) the system (3.7) is satisfied, and

(b) if $h \in \mathcal{H} \backslash\{0\}$ is such that $\left\langle Q_{i} \bar{x}+c_{i}, h\right\rangle=0, i \in I_{1}(\bar{x}),\left\langle Q_{i} \bar{x}+c_{i}, h\right\rangle \leq 0, i \in I_{2}(\bar{x})$, where $I_{1}(\bar{x})=\left\{i: g_{i}(\bar{x})=0, \lambda_{i}>0\right\}, I_{2}(\bar{x})=\left\{i: g_{i}(\bar{x})=0, \lambda_{i}=0\right\}$, then $\langle h, Q h\rangle>0$.

The proof of this theorem is similar to that of Theorem 4.4 so it is omitted here. 


\section{Conclusions}

In this paper we consider quadratic programming problems in Hilbert spaces and propose condition for a feasible point to be (a strict) a local solution of quadratic programming problems whose constraint set is defined by finitely many convex quadratic inequalities in Hilbert spaces. Our result is established without requesting finiteness dimension of constraint set.

\section{Acknowledgments}

This research is funded by Vietnam National Foundation for Science and Technology Development (NAFOSTED) under grant number 101.01-2018.306. The author was supported by the Hanoi University of Industry [grant number 37-2020-RD]. The author would like to thank Prof. Nguyen Dong Yen for comments that greatly improved the paper. The author would like to thank Prof. Nguyen Nang Tam for valuable suggestions. The author would like to express his sincere thanks to the anonymous referees and editors for insightful comments and useful suggestions.

\section{References}

[1] J. F. Bonnans and A. Shapiro, Perturbation Analysis of Optimization Problems, Springer Series in Operations Research. Springer-Verlag, New York, 2000.

[2] J. M. Borwein, Necessary and sufficient conditions for quadratic minimality, Numer. Funct. Anal. Optim. 5 (1982), no. 2, 127-140.

[3] V. V. Dong and N. N. Tam, On the solution existence of convex quadratic programming problems in Hilbert spaces, Taiwanese J. Math. 20 (2016), no. 6, 1417-1436.

[4] - On the solution existence of nonconvex quadratic programming problems in Hilbert spaces, Acta Math. Vietnam 43 (2018), no. 1, 155-174.

[5] A. D. Ioffe, Regular points of Lipschitz functions, Trans. Amer. Math. Soc. 251 (1979), 61-69.

[6] A. D. Ioffe and V. M. Tihomirov, Theory of Extremal Problems, Studies in Mathematics and its Applications 6, North-Holland, Amsterdam, 1979.

[7] D. S. Kim, N. N. Tam and N. D. Yen, Solution existence and stability of quadratically constrained convex quadratic programs, Optim. Lett. 6 (2012), no. 2, 363-373. 
[8] G. M. Lee, N. N. Tam and N. D. Yen, Quadratic Programming and Affine Variational Inequalities: A qualitative study, Nonconvex Optimization and its Applications 78, Springer-Verlag, New York, 2005.

[9] H. Maurer and J. Zowe, First and second order necessary and sufficient optimality conditions for infinite-dimensional programming problems, Math. Programming 16 (1979), no. 1, 98-110.

[10] I. E. Schochetman, R. L. Smith and S. K. Tsui, Solution existence for infinite quadratic programming, in: Mathematical Programming with Data Perturbations, 363-385, Lecture Notes in Pure and Appl. Math. 195, Dekker, New York, 1998.

[11] K. C. Sivakumar and J. Mercy Swarna, Explicit solutions of infinite quadratic programs, J. Appl. Math. Comput. 12 (2003), no. 1-2, 211-218.

[12] N. N. Tam and T. V. Nghi, On the solution existence and stability of quadratically constrained nonconvex quadratic programs, Optim. Lett. 12 (2018), no. 5, 1045-1063.

Vu Van Dong

Hanoi University of Industry, Vietnam

E-mail addresses: dongvv@haui.edu.vn, vuvdong@gmail.com 